\title{
Wide-Area Measurement-Based Voltage Stability Sensitivity and its Application in Voltage Control
}

\author{
Houhe Chen ${ }^{1}$, Tao Jiang ${ }^{1 *}$, Haoyu Yuan ${ }^{2}$, Hongjie $\mathrm{Jia}^{3}$, Linquan $\mathrm{Bai}^{2}$ and Fangxing $\mathrm{Li}^{2 *}$ \\ ${ }^{1}$ : Department of Electrical Engineering, Northeast Electric Power University, Jilin, Jilin 132012, China. \\ ${ }^{2}$ : Electrical Engineering and Computer Science Dept., University of Tennessee, Knoxville, TN 37996, USA. \\ ${ }^{3}$ : Key Laboratory of Smart Grid of Ministry of Education, Tianjin University, Tianjin 300072, China. \\ *: Contact author: tjiang@ nedu.edu.cn; fli6@utk.edu.
}

Abstract - This work proposes a measurement-based voltage stability index, namely wide-area measurement-based voltage stability sensitivity based on $L$ index. The sensitivities of $L$ index with respect to nodal reactive power $(\mathrm{Q})$ and real power $(\mathrm{P})$ injections are first derived. The derived $L-Q$ and $L-P$ sensitivities analyze the impact of nodal injection to nodal voltage stability and can help determine the reactive power compensation and emergency load shedding amount for voltage stability control. To improve the computational efficiency, a simplified $L$ index, $L^{\prime}$, along with its sensitivities with respect to nodal reactive and real power injection ( $L^{\prime}-Q$ and $L^{\prime}-P$ sensitivities) are derived which makes the proposed approach suitable for the practical large-scale systems. Moreover, a control strategy for voltage stability is proposed based on the $L-Q, L-P, L^{\prime}-Q$, and $L^{\prime}-P$ sensitivities. The proposed sensitivities and control strategy are tested on the New England 39 bus system and the IEEE 118 system. Test results on both systems verify the proposed sensitivities and the control strategy by demonstrating their accuracy and robustness in voltage stability assessment and control. In conclusion, the proposed measurement-based sensitivities can be applied to voltage stability assessment and control by using the wide-area measurements.

Keywords - Wide area measurement systems (WAMS), voltage stability, $L$ index, sensitivity analysis, voltage stability control, reactive compensation, and emergency load shedding.

\section{Introduction}

In large scale interconnected power systems, increasing penetration of renewable energy and ever growing demand have challenged the systems to operate closer to their voltage stability limits [1]-[8]. Consequently, voltage stability assessment (VSA) and control have received increasing attention [9]-[13].

Indeed, voltage stability has always been a major concern in modern power systems and has been extensively explored in the literature. In VSA, various voltage stability indices (VSIs) have been proposed for analyzing and monitoring the system stability [13]. Theses VSIs include the voltage stability factor (VSF) [14], loading margin 
[15], smallest eigenvalue of Jacobian matrix [16], voltage instability proximity index (VIPI) based on saddle-node bifurcation (SNB) [17], voltage controllability index (VCI) [18], local loading margin index (PLmgn) [19], closest loadability limit (CLL) based on multiple load flow solutions [20], etc. These indices have been widely used in VSA applications for assessing voltage stability and their sensitivities have been studied to evaluate impacts of parameter change on VSA. However, the aforementioned indices are all based on the power flow model of the system, which makes their accuracy highly dependable on the accuracy of the system model. In fact, models and parameters of a practical power system are updated by an energy management system (EMS) whose accuracy is affected by measurement noise, errors, and other uncertain factors. Moreover, the computational costs of these power-flow-based indices and their sensitivities increase drastically as the system scales up. Consequently, these model-based indices and sensitivities are mostly applied in system planning and other offline studies rather than in online applications.

Recently, the development of wide-area measurement systems (WAMS) has provided alternative VSA approaches to traditional model-based ones. Aided by WAMS, such measurement-based VSAs have become an important research area [21]-[35]. In [23], an online voltage security assessment based on a decision tree that is trained with offline simulation but makes decisions on phasor measurement unit (PMU) measurements has been proposed. In [24], an online VSA approach for system status monitoring and voltage collapse prediction is proposed through calculating the line stability index. In [25], a local voltage stability index based on Tellegen's theorem is proposed. In [26], by analyzing the voltage deviation of two consecutive measurements, a new online voltage stability index is proposed. In [27], based on the Thevenin equivalent circuit, an online VSA approach assessing three types of transfer margins (real, reactive, and apparent power) is proposed. In [28], a local voltage stability index, $L$ index, with the range between 0 (no load) and 1 (voltage collapse) is proposed. Based on the $L$ index of [28], an improved $L$ index incorporating ZIP load model (ZIP load is a combination of constant-impedance, constant-current, and constant-power load component) is proposed in [29]. In [30], a hybrid approach which is capable of assessing voltage stability for $\mathrm{N}-1$ contingency is proposed. VSA approaches introduced by [24]-[30] are all based on Thevenin equivalent circuit of the power system. Theoretically, within a short time period, the outside system supplying one particular load bus can be represented by the two-bus Thevenin circuit. In [31], three consecutive measurements of voltage and current from PMU are used to correct the phase drifts caused by the measurement slip frequency. Although, in theory, the outside system can be equivalent into a Thevenin circuit, such strong simplification will lose important information or characteristics of the highly nonlinear power system. 
If deployed online, measurement-based VSA approaches introduced in [21]-[35] are capable of increasing the situational awareness of the system operators by providing fast real-time assessment of the voltage stability. However, the approaches based on the Thevenin circuit, which is essentially a black box model, provide little analytical information of the system besides the one dimensional stable or instable information. Moreover, the existing works did not provide sensitivity analyses on the parameters of the Thevenin circuits, which are crucial for voltage stability prediction and control. From the view point of system operators, the information regarding which controls to take when facing the situation of voltage instability is equally important as the awareness of the instability. Clearly, in the existing works, there is significant effort regarding the latter issue, which focuses on improving the efficiency and accuracy of the measurement-based VSA [32]-[34], but little attention paid to the former issue of voltage stability prediction or control.

With such motivation, based on [28] and [29], this work provides the sensitivity analyses of the $L$ index through its differential equation. The sensitivities of $L$ index to nodal reactive and real power injection, i.e. $L-Q$ and $L-P$ sensitivities, are derived in order to investigate the impact of nodal injection on the voltage stability margin. Then, a simplified $L$ index, $L^{\prime}$ and its sensitivities to nodal reactive and real power injection, $L^{\prime}-Q$ and $L^{\prime}-P$ sensitivities, have been proposed based on the same assumption made when deriving the DC power flow for transmission level. The $L^{\prime}$ index as well as the $L^{\prime}-Q$ and $L^{\prime}-P$ sensitivities are highly computational efficiencies and are suitable for large-scale interconnected power systems. Moreover, enabled by the proposed sensitivities, a voltage stability control strategy is proposed, which provides the system operator with effective control decisions on reactive power compensation and load shedding. Finally, the proposed sensitivities and control strategy are verified and analyzed on the New England 39-bus system and IEEE 118 bus system.

\section{Voltage Stability Index and Its Simplification}

\subsection{Voltage Stability Index and Sensitivities}

Several approaches of PMU-based voltage stability assessment have been proposed in the literature and are compared in [35]. This work is based on the coupled single-port circuit (CSPC) concept proposed in [11]. The power system can be modeled as a multi-port network. All the generators and load buses are brought outside of the network. And the branches and the tie buses, the buses with no current injection to, are modeled inside the network. According to Kirchhoff's current law (KCL) in bulk power systems, the nodal current injections vector can be expressed as: 


$$
\left[\begin{array}{l}
\boldsymbol{I}_{\boldsymbol{G}} \\
\boldsymbol{I}_{\boldsymbol{L}} \\
0
\end{array}\right]=\left[\begin{array}{lll}
\boldsymbol{Y}_{G G}^{\prime} & \boldsymbol{Y}_{G L}^{\prime} & \boldsymbol{Y}_{G K}^{\prime} \\
\boldsymbol{Y}_{L G}^{\prime} & \boldsymbol{Y}_{L L}^{\prime} & \boldsymbol{Y}_{L K}^{\prime} \\
\boldsymbol{Y}_{K G}^{\prime} & \boldsymbol{Y}_{K L}^{\prime} & \boldsymbol{Y}_{K K}^{\prime}
\end{array}\right]\left[\begin{array}{l}
\boldsymbol{V}_{\boldsymbol{G}} \\
\boldsymbol{V}_{\boldsymbol{L}} \\
\boldsymbol{V}_{\boldsymbol{K}}
\end{array}\right]
$$

where $\boldsymbol{V}_{G}$ and $\boldsymbol{I}_{G}$ are voltage and current vectors of generator buses; $\boldsymbol{V}_{L}$ and $\boldsymbol{I}_{L}$ are voltage and current vectors of load buses; $\boldsymbol{V}_{\boldsymbol{K}}$ is voltage vector of interconnecting buses where no load or generator is attached to; $Y_{G G}^{\prime}, Y_{G L}^{\prime}, Y_{G K}^{\prime}, Y_{L G}^{\prime}, Y_{L L}^{\prime}, Y_{L K}^{\prime}, Y_{K G}^{\prime}, Y_{K L}^{\prime}$, and $Y_{K K}^{\prime}$ are corresponding submatrices of the system admittance matrix.

Eliminating the interconnecting buses, two types of buses are remained: the set of generator buses, $\boldsymbol{\alpha}_{G}$, and the set of load buses, $\boldsymbol{\alpha}_{L}$. The system in (1) can be further expressed by

$$
\left[\begin{array}{c}
I_{G} \\
I_{L}
\end{array}\right]=\left[\begin{array}{ll}
Y_{G G} & Y_{G L} \\
Y_{L G} & Y_{L L}
\end{array}\right]\left[\begin{array}{c}
V_{G} \\
V_{L}
\end{array}\right]
$$

where $Y_{G G}=Y_{G G}^{\prime}-Y_{G K}^{\prime} Y_{K K}^{\prime-1} Y_{K G}^{\prime}, Y_{G L}=Y_{G L}^{\prime}-Y_{G K}^{\prime} Y_{K K}^{\prime-1} Y_{K L}^{\prime}, Y_{L G}=Y_{L G}^{\prime}-Y_{L K}^{\prime} Y_{K K}^{\prime-1} Y_{K G}^{\prime}, Y_{L L}=Y_{L L}^{\prime}-Y_{L K}^{\prime} Y_{K K}^{\prime-1} Y_{K L}^{\prime}$.

Let $\boldsymbol{Z}_{L L}=\boldsymbol{Y}_{L L}^{-1}$, (2) can be expressed by

$$
\left[\begin{array}{l}
I_{G} \\
V_{L}
\end{array}\right]=\left[\begin{array}{ll}
Y_{G G}-Y_{G L} Z_{L L} Y_{L G} & Y_{G L} Z_{L L} \\
-Z_{L L} Y_{L G} & Z_{L L}
\end{array}\right]\left[\begin{array}{c}
V_{G} \\
I_{L}
\end{array}\right]
$$

From (3), the terminal voltage of a load bus can be expressed by

$$
V_{L}=Z_{L L} I_{L}+Z_{L G} V_{G}
$$

where $\boldsymbol{Z}_{L G}=-\boldsymbol{Z}_{\boldsymbol{L} L} \boldsymbol{Y}_{\boldsymbol{L G}}$.

According to (4), the detailed expression of voltage at load bus $j, \dot{V}_{L j}$, is

$$
\dot{V}_{L j}=Z_{L L j j} \dot{I}_{L j}+\sum_{\substack{i \in \alpha_{L} \\ i \neq j}} Z_{L L j i} \dot{I}_{L i}+\sum_{k \in \alpha_{G}} Z_{L G j k} \dot{V}_{G k}
$$

where $\dot{I}_{L j}$ and $\dot{I}_{L i}$ is the current injection at load bus $j$ and $i$ respectively; $Z_{L L j j}$ is the self-impedance of load bus $j$; $Z_{L L j i}$ is the coupling impedance between bus $j$ and $i ; Z_{L G j k}$ is coupling impedance between load bus $j$ and generator bus $k$; and $\dot{V}_{G k}$ is the terminal voltage at generator bus $k$.

Express $\dot{V}_{L j}$ in (5) in terms of a combination of a voltage source $\dot{V}_{e q j}$ and current source $\dot{I}_{e q j}$ :

$$
\dot{V}_{L j}=Z_{e q j} \dot{I}_{L e q j}+\dot{V}_{e q j}
$$

where 


$$
\begin{gathered}
\dot{I}_{e q j}=\dot{I}_{L j}+\sum_{\substack{i \in \alpha_{L} \\
i \neq j}} \frac{Z_{L L j i}}{Z_{e q j}} \dot{I}_{L i} \\
\dot{V}_{e q j}=-\sum_{k \in \alpha_{G}} Z_{L G j k} \dot{V}_{G k} \\
Z_{e q j}=Z_{L L j j}
\end{gathered}
$$

Multiplying $\dot{V}_{L j}^{*}$ to both side of (6) yields

$$
V_{L j}^{2}+\dot{V}_{e q j}\left(\dot{V}_{L j}\right) *=Z_{e q j} \dot{I}_{e q j}\left(\dot{V}_{L j}\right) *
$$

Define the equivalent apparent power at load bus $j$ as $\dot{S}_{e q j}=\dot{V}_{L j}\left(\dot{I}_{e q j}\right) *$. With $Y_{L L j j}=1 / Z_{L L j j}$, (10) can be further expressed as

$$
V_{L j}^{2}+\dot{V}_{e q j}\left(\dot{V}_{L j}\right) *=\left(\dot{S}_{e q j}\right) * / Y_{L L j j}
$$

Based on (11), the voltage stability index for load bus $j, L_{j}$, proposed in [28] and [29], $L_{j}$, can be represented as follows:

$$
L_{j}=\frac{1}{V_{j}^{2}}\left|\sum_{i \in \alpha_{L}} \frac{Z_{j i}^{*} \tilde{S}_{i} \dot{V}_{j}}{\dot{V}_{i}}\right|
$$

where $\dot{V}_{i}$ and $\dot{V}_{j}$ are the voltage phasors at Bus $i$ and $j ; V_{j}$ is the magnitude of voltage at Bus $j ; S_{i}$ is the apparent power of load bus $i ; Z_{L L, j i}$ is a coupling impedance between load buses $j$ and $i[25] ; \alpha_{L}$ is the set of load buses.

$L_{j}$ in (12) can be expressed with more detail:

$$
\begin{gathered}
L_{j}=\frac{1}{V_{j}^{2}} \sqrt{f^{2}+g^{2}} \\
\left\{\begin{array}{l}
f_{i}=P_{i}\left(R_{i j} \cos \theta_{i j}-X_{i j} \sin \theta_{i j}\right)+Q_{i}\left(X_{i j} \cos \theta_{i j}+R_{i j} \sin \theta_{i j}\right) \\
f=\sum_{i \in \alpha_{L}} \frac{V_{j}}{V_{i}} f_{i} \\
g_{i}=Q_{i}\left(R_{i j} \cos \theta_{i j}-X_{i j} \sin \theta_{i j}\right)-P_{i}\left(X_{i j} \cos \theta_{i j}+R_{i j} \sin \theta_{i j}\right) \\
g=\sum_{i \in \alpha_{L}} \frac{V_{j}}{V_{i}} g_{i}
\end{array}\right.
\end{gathered}
$$

where $P_{i}$ and $Q_{i}$ are the nodal real and reactive power injection at load bus $i$; $\theta_{i j}$ is the phase angle difference of voltages at load bus $i$ and bus $j ; R_{i j}$ and $X_{i j}$ are coupling resistance and reactance between load bus $i$ and $j$.

The $L$ indices of all load buses construct an index vector $L=\left[L_{1}, L_{2}, \ldots, L_{n}\right]$ for the whole system, where $n \in \boldsymbol{\alpha}_{L}$. The index of VSA for the whole system can be defined as: 


$$
L=\|L\|_{\infty}
$$

The voltage stability of the system can be determined by the local voltage stability index ( $L$ index) [28], [29]:

1) $L<1.0$, system is voltage stable, 2) $L=1.0$, system is critical, 3) $L>1.0$, system is voltage instable.

Based on (13) and (15), the voltage stability of each load bus as well as that of the whole system can be assessed. However, no further information that can be used to guide the decisions of following control actions is available. To provide such crucial information, sensitivity analyses on the voltage stability index are performed in this work to investigate the impact of nodal power injection change on voltage stability. With the proposed sensitivities, the system operators will be able to take the most sensitive control actions that are available to prevent or correct voltage instability.

Based on (13), the exact differential equation of the $L_{j}$ index of load bus $j$ can be expressed as

$$
\Delta L_{j}=\frac{\partial L_{j}}{\partial Q} \Delta Q+\frac{\partial L_{j}}{\partial P} \Delta P+\frac{\partial L_{j}}{\partial \theta} \Delta \theta+\frac{\partial L_{j}}{\partial V} \Delta V
$$

The partial derivatives in (16) can be derived based on (14) as

$$
\begin{aligned}
& \frac{\partial L_{j}}{\partial P_{i}}=\frac{\left\lfloor\left(R_{i j} \cos \theta_{i j}-X_{i j} \sin \theta_{i j}\right) f\right\rfloor-\left\lfloor\left(X_{i j} \cos \theta_{i j}+R_{i j} \sin \theta_{i j}\right) g\right\rfloor}{V_{j}^{2} V_{i} L_{j}} \\
& \frac{\partial L_{j}}{\partial Q_{i}}=\frac{\left\lfloor\left(X_{i j} \cos \theta_{i j}+R_{i j} \sin \theta_{i j}\right) f\right\rfloor+\left\lfloor\left(R_{i j} \cos \theta_{i j}-X_{i j} \sin \theta_{i j}\right) g\right\rfloor}{V_{j}^{2} V_{i} L_{j}} \\
& \frac{\partial L_{j}}{\partial \theta_{i j}}=\frac{g_{i} f-f_{i} g}{V_{j}^{2} V_{i} L_{j}} \\
& \left\{\begin{array}{l}
\frac{\partial L_{j}}{\partial V_{i}}=-\frac{\left(f_{i} \cdot f+g_{i} \cdot g\right)}{V_{i}^{2} V_{j}^{2} L_{j}} \\
\frac{\partial L_{j}}{\partial V_{j}}=-\frac{\left(f_{j} \cdot f+g_{j} \cdot g\right)+V_{j}^{3} L_{j}^{2}}{V_{j}^{4} L_{j}}
\end{array}\right.
\end{aligned}
$$

According to (16), the $L$ index is mainly affected by the $P, Q, \theta$, and $V$ of the load buses. Since $\theta$ and $V$ of load buses are dependent on nodal injection $P$ and $Q$, the dominant factors that impact the voltage stability are $P$ and $Q$. When the system is at a stable operating point, the impact of the variation of real and reactive power injection $P$ and $Q$ of load bus to the voltage stability can then be investigated through (21).

$$
\Delta L_{j}=\left[\frac{\partial L_{j}}{\partial P_{1}}, \frac{\partial L_{j}}{\partial P_{2}}, \cdots \frac{\partial L_{j}}{\partial P_{n}}\right]\left[\Delta P_{1}, \Delta P_{2}, \cdots, \Delta P_{n}\right]^{T}+\left[\frac{\partial L_{j}}{\partial Q_{1}}, \frac{\partial L_{j}}{\partial Q_{2}}, \cdots \frac{\partial L_{j}}{\partial Q_{n}}\right]\left[\Delta Q_{1}, \Delta Q_{2}, \cdots, \Delta Q_{n}\right]^{T}
$$

From (21), it is seen that the variation of the nodal injection of a load bus will not only affect the voltage stability ( $L$ index) of that bus, but also the stability of other buses. Expressed in (16)-(21), the $L-P$ and $L-Q$ 
sensitivities can be used to analyze the coupling effect of the load buses in VSA and to quantitatively estimate the impact of variation of nodal real and reactive injections to voltage stability.

\subsection{Simplified Voltage Stability Index and Sensitivities}

According to (13) to (21), the calculation of $L$ index, $L-P$, and $L-Q$ sensitivities for all load buses involves non-trivial computation effort especially when the system scale increases. In high-voltage transmission networks, it is a common practice to simplify the calculation and to improve the computational efficiency by taking advantage of the following two characteristics: 1) the line reactance is much greater than line resistance and 2) the angle difference between two nearby voltage phasors is close to 0. By neglecting the line resistance and assuming the angle difference close to 0 , the $L$ index in (13) and (14) can be simplified as $L^{\prime}$ index:

$$
\begin{aligned}
& L_{j}^{\prime}=\frac{1}{V_{j}^{2}} \sqrt{f^{\prime 2}+g^{\prime 2}} \\
& \left\{\begin{array}{l}
f_{i}^{\prime}=Q_{i} X_{i j} \\
f^{\prime}=\sum_{i \in \alpha_{L}} \frac{V_{j}}{V_{i}} f_{i}^{\prime} \\
g_{i}^{\prime}=-P_{i} X_{i j} \\
g^{\prime}=\sum_{i \in \alpha_{L}} \frac{V_{j}}{V_{i}} g_{i}
\end{array}\right.
\end{aligned}
$$

Note that the second assumption is strict as angle difference may increase as load level increases. However, in our test, it is demonstrated that the simplified $L^{\prime}$ index is very close to $L$ index even at the voltage collapsing point. Therefore, the simplified $L^{\prime}$ index is proposed as an engineering practice for situations where the computation of the complex $L$ index is not practical.

Comparing (22) with (14), in the simplified index, the real power and reactive power are decoupled into $f^{\prime}$ and $g$ ' respectively and the computation burden can be significantly reduced.

Following (22) and (23), the $L-Q$ sensitivities in (17) can hereby be simplified as $L^{\prime}-Q$ sensitivities:

$$
\frac{\partial L_{j}^{\prime}}{\partial Q_{i}}=\frac{X_{L L i j} \sum_{i \in \alpha_{L}} \frac{Q_{i} X_{L L i j}}{V_{i}}}{V_{j} V_{i} \sqrt{\left(\sum_{i \in \alpha_{L}} \frac{Q_{i} X_{L L i j}}{V_{i}}\right)^{2}+\left(\sum_{i \in \alpha_{L}} \frac{-P_{i} X_{L L i j}}{V_{i}}\right)^{2}}}
$$

Similar to (24), the $L-P$ sensitivities in (18) can hereby be simplified as $L^{\prime}-P$ sensitivities: 


$$
\frac{\partial L_{j}^{\prime}}{\partial P_{i}}=\frac{X_{L L i j} \sum_{i \in \alpha_{L}} \frac{P_{i} X_{L L i j}}{V_{i}}}{V_{j} V_{i} \sqrt{\left(\sum_{i \in \alpha_{L}} \frac{Q_{i} X_{L L i j}}{V_{i}}\right)^{2}+\left(\sum_{i \in \alpha_{L}} \frac{-P_{i^{2}} X_{L L i j}}{V_{i}}\right)^{2}}}
$$

\subsection{Interpretation and Application of the Sensitivities}

From (17), (18), (24), and (25), it is found that the $L-Q, L-P, L^{\prime}-Q$ and $L^{\prime}-Q$ sensitivities are greater than 0 , which means the $L$ index or $L^{\prime}$ index will increase (indicates less voltage stable) as the nodal reactive or real power injection (load) increases. This can be explained that when the reactive/real power that flows into one load bus increases, the reactive/real power losses as well as the voltage drops over the network near this load bus increase accordingly, which in turn makes the load bus less voltage stable. Meanwhile, the voltage stability of one bus is affected not only by its own reactive/real power injection but also by reactive/real power injections at other buses. This is because the deficiency of reactive support at one bus will lead to reactive power transfer from neighboring buses through an interconnected network, which eventually will lead to reduced voltage stability at the neighboring buses.

The major contribution of this work is that the proposed $L-Q, L-P, L^{\prime}-Q$ and $L^{\prime}-Q$ sensitivities provide measurement-based tools for quantitatively analyzing and estimating the impact of nodal reactive and real injection variation on voltage stability. The proposed sensitivities give a comprehensive analysis of not only how other load buses affect the voltage stability of one particular load bus but also how the particular load bus affects the voltage stability of other load buses in the network. Therefore, system operators will be able to make reasonable and effective control decisions to improve voltage stability and will benefit from the comprehensive system-level information about voltage stability coupling among load buses supported by the proposed sensitivities. Following this path, to explore the utility of the proposed sensitivities, a control strategy for improving system-level voltage stability through reactive compensation and emergency load shedding is proposed.

Let $L_{0}$ be the threshold of $L$ index that system must operate below. When $L_{i}$, the $L$ index of any load bus $i$, is beyond $L_{0}$, corresponding control actions have to be taken to bring the $L$ index below $L_{0}$. According to the availability of the reactive reserve in bus $i$ and the rest of the system, three control strategies are proposed:

(1) Reactive compensation at Bus $i$. When there is sufficient reactive reserve at Bus $i$, reactive compensation is directly applied on Bus $i$ and the amount to be compensated is determined by: 


$$
\Delta Q_{i}=\frac{\left(L_{i}-L_{i 0}\right)}{L Q_{i i}}
$$

where $\Delta Q_{i}$ is the amount to be compensated at Bus $i ; L_{i}$ is the $L$ index of Bus $i$ before compensation; $L_{i 0}$ is the threshold $L$ index for bus $i$; and $L Q_{i i}$ is the sensitivity of $L_{i}$ to $Q_{i}$.

(2) Reactive compensation at Bus $j(j \neq i)$. When there is not sufficient reactive reserve at Bus $i$, which means $\Delta Q_{i}$ determined by (26) is larger than the reactive reserve at Bus $i$, all of the reserve at Bus $i$ is first applied. Then reactive compensation at the Bus $j$ which has the largest sensitivities to $L_{i}$ is applied at the amount determined by:

$$
\Delta Q_{j}=\frac{\left(L_{i}-L_{i 0}-\Delta Q_{i 0} L Q_{i i}\right)}{L Q_{i j}}
$$

where $\Delta Q_{j}$ is the amount to be compensated at Bus $j ; \Delta Q_{i 0}$ is the available amount of reactive reserve at Bus $j$; and $L Q_{i j}$ is the sensitivity of $L_{i}$ to $Q_{j}$.

(3) Load shedding. In emergency conditions such as N-1 contingency, load shedding should be applied when $L_{0}$ is not met. The amount of load shedding can be determined by:

$$
\begin{aligned}
\Delta P_{i} & =\frac{L_{i}-L_{i 0}}{L P_{i i}+\tan \varphi_{i} L Q_{i i}} \\
\Delta Q_{i} & =\tan \varphi_{i} \Delta P_{i}
\end{aligned}
$$

where $\Delta P_{i}$ and $\Delta Q_{i}$ are the amount of real and reactive power load to be shed at Bus $i ; L P_{i i}$ is the sensitivity of $L_{i}$ to $P_{i} ; L Q_{i i}$ is the sensitivity of $L_{i}$ to $Q_{i}$; and $\cos \varphi_{i}$ is the power factor of load at Bus $i$. Through (28), it is guaranteed that real power and reactive power load are shed at the same power factor of the original load.

Since voltage instability is usually observed at several buses, when there are multiple locations that violate the thresholds, the control actions for each bus should be determined similarly according to (26) through (28) in order to maintain the stability of the whole system.

\section{4. $L-Q\left(\right.$ or $\left.L^{\prime}-Q\right)$ Sensitivities on Special Buses}

When deriving the $L$ index and the $L-Q$ sensitivity in Section 2.1, the buses in the network are clustered into generator, tie-line, and load groups to calculate $\boldsymbol{Z}_{L L}$ [33]. During the realistic operation of the power system, it is possible to transit one type of bus to another. Therefore, the classification of special buses and the transition of certain buses need to be addressed.

(1) Buses installed with reactive compensation devices. The interconnecting buses (or tie-line buses) are defined as buses without connected loads or generators [33]. In practice, there are cases where reactive compensation devices are installed on such interconnecting buses. If there are fixed capacitors installed, the 
interconnecting buses are still considered as interconnecting buses and the capacitors are modelled into the shunt impedance. If the installed devices are synchronous condensers, static VAR compensators (SVC), or static synchronous compensators (STATCOM), these buses are modelled as PV bus and should be clustered into generator groups before the reactive compensation reaches the limit. After the compensation limit has been reached, these buses should be clustered into the load group.

(2) Generators with no more reactive compensation capability. When the excitation current of a generator reaches its maximum, the generator is no longer able to provide reactive compensation in order to maintain the generator terminal voltage. In power flow model, this bus should transit from PV bus to PQ bus. In our study, this bus should be clustered into load group after the transition. Also, when a generator is disconnected from the grid, the bus the generator used to connect to should be treated as the interconnecting bus.

\section{Implementation of the Proposed Method}

The steps to implement the proposed voltage stability index, sensitivities and control strategies are:

Step 1: Referring to [28] and [29], form the network impedance matrix $\boldsymbol{Z}_{\boldsymbol{L} L}$ according to the types of nodes in the network and the system impedance matrix.

Step 2: Based the measurements of voltage, current, real and reactive power from WAMS, calculate the $L$ or $L^{\prime}$ index of every load bus according to (13) and (22). Determine the voltage critical bus and assess the system-level voltage stability by finding the largest $L$ or $L^{\prime}$ index of all load buses.

Step 3: Determine whether or not the voltage stability index crosses the threshold. If the index is within the threshold, go to Step 2; if the index is beyond the threshold (instable or insecure), go to Step 4.

Step 4: Calculate the $L-Q$ and $L-P$ or $L^{\prime}-Q$ and $L^{\prime}-P$ sensitivities of the buses with indices beyond the threshold according to (17) (18) or (24) (25).

Step 5: Determine and apply the control actions according to (26)-(28) based on the reactive reserve capability and the sensitivities calculated in Step 4.

Step 6: After the controls are applied, check if the critical bus determined in Step 2 is still the critical bus. If there is a shift of critical bus, go to Step 2.

One thing to notice is that it is important to track the system topology change and the bus type transition as the system operates to achieve correct voltage stability assessment and control. When such situation occurs, the system information should be updated and the application should go back to Step 1 to calculate the updated $\boldsymbol{Z}_{\boldsymbol{L} L}$ and then go through the following steps for an updated voltage stability assessment and control.

Also, in Step 6, an outer loop is added in order to guarantee all load buses are properly controlled. 
For the proposed method to be practically implemented in the field, pre-processing of measurements to exclude noise or errors is required. In order to focus on the proposed methods within limited length, noise and errors are not modelled in this work.

\section{Case Study}

In order to verify the applicability and effectiveness of the proposed methods, case studies on New England 39-bus system and IEEE 118 bus system are performed and demonstrated in this section. Existing measurement-based voltage stability sensitivity or control strategies are very limited. A recent work [12] proposes real-time wide-area loading margin sensitivity for reactive under normal operating condition. In our study, the proposed approach achieves comparable results but do extend the measurement-based sensitivity to real power and emergency load shedding. Therefore, detailed comparison with other approaches are not provided for the succinct of the work.

\subsection{New England 39-bus system}

In the case study, the PV curve of the New England 39-bus system [36] with all the loads increasing evenly is calculated through Continuation Power Flow (CPF). The $L$ index and $L^{\prime}$ index as well as the sensitivities are calculated based on the operation points on the PV curve which are essentially power flow results.

\subsubsection{Verification of $L^{\prime}$ Index}

$L^{\prime}$ index is a simplified form of $L$ index. In order to justify the two assumptions made when deriving the $L^{\prime}$ index, the $L^{\prime}$ indices of all load buses are benchmarked with the corresponding $L$ indices on the 39-bus system for all of the operating points on the PV curves. The $L$ indices and $L^{\prime}$ indices are depicted in Fig. 1 .

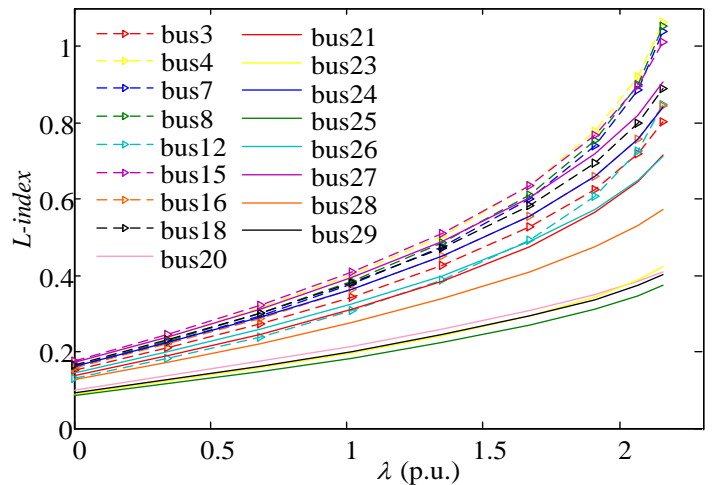

(a) $L$ indices

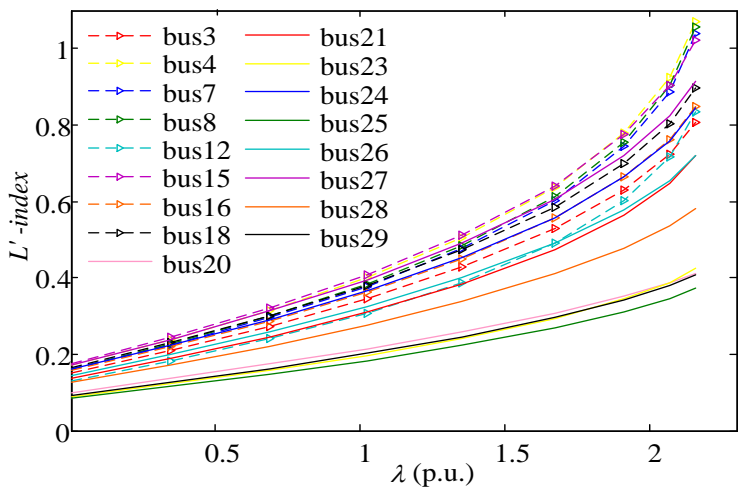

(b) $L^{\prime}$ indices

Fig. 1. $L$ and $L^{\prime}$ indices for all buses on all operating points

Comparing Fig. 1(a) and Fig. 1(b), the $L$ indices and $L^{\prime}$ indices have the same trends and both indicate instability at the last operating point. Moreover, the relative errors of the $L^{\prime}$ indices in Fig. 1(b) compared with the 
corresponding $L$ indices in Fig. 1(a) are summarized in Fig. 2. In Fig. 2, it is seen that as the load increases $(\lambda$ increases) the relative errors of $L^{\prime}$ tend to increase. The largest errors of all buses (Bus 15) increase from $0.3850 \%$ at the base case to $1.8276 \%$ at the critical load level. However, the largest error is still under $2 \%$ and can be considered relatively small in VSA applications. From the results shown in Fig. 1 and Fig. 2, it is evidenced that the simplified $L^{\prime}$ index provides reasonable accuracy for VSA applications.

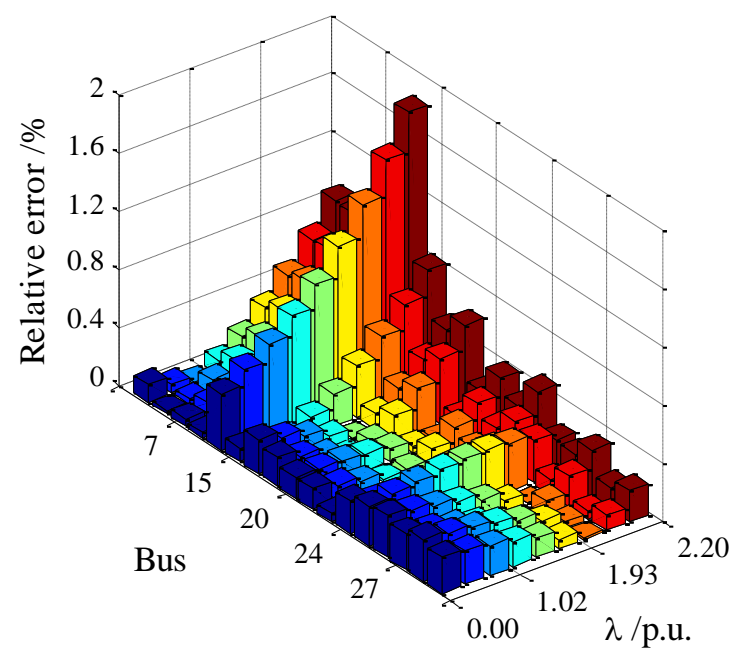

Fig. 2. Relative errors of $L^{\prime}$ indices compared with $L$ indices

\subsubsection{Verification of $L-Q, L-P, L^{\prime}-Q$ and $L^{\prime}-P$ Sensitivities}

Upon verification of the $L^{\prime}$ index, the proposed $L-Q, L-P, L^{\prime}-Q$ and $L^{\prime}-P$ sensitivities are verified. The $L$ and $L^{\prime}$ indices of all load buses under the base load condition are illustrated in TABLE 1 . According to the table, Bus 15 is the critical bus with the highest indices: an $L$ index of 0.2005 and an $L^{\prime}$ index of 0.2009 . The $L-Q$ and $L^{\prime}-Q$ sensitivities are calculated and demonstrated in Fig. 3. The $L-P$ and $L^{\prime}-P$ sensitivities are calculated and demonstrated in Fig. 4. It is seen in Fig. 3 and Fig. 4 that all the sensitivities are positive indicating that increase in nodal reactive or real load will decrease the voltage stability monotonically. It is also shown that the diagonal elements in both sensitivity matrices are dominant which means that the variation of reactive or real power at a particular bus has a higher impact on its own $L$ or $L^{\prime}$ index than the variation of reactive or real power at other buses. The reason for this is that the variation of reactive or real power injection at a certain bus casts its impact on other buses by affecting the power flow across the network while the variation at its own location can directly cast such impact without any reactive or real losses across the network. From this case study, it is shown that voltage stability is a local problem in which the coupling of the VSA of a bus to its own reactive or real power injection is the strongest. 
Table $1 L$ and $L^{\prime}$ Indices under Base Load Condition

\begin{tabular}{ccc||ccc}
\hline Bus No & $\boldsymbol{L}$ index & $\boldsymbol{L}^{\prime}$ index & Bus No & $\boldsymbol{L}$ index & $\boldsymbol{L}^{\prime}$ index \\
\hline 3 & 0.1697 & 0.1699 & 21 & 0.1532 & 0.1530 \\
4 & 0.1973 & 0.1976 & 23 & 0.0976 & 0.0974 \\
7 & 0.1856 & 0.1859 & 24 & 0.1799 & 0.1796 \\
8 & 0.1889 & 0.1892 & 25 & 0.0909 & 0.0905 \\
12 & 0.1511 & 0.1511 & 26 & 0.1602 & 0.1602 \\
$\mathbf{1 5}$ & $\mathbf{0 . 2 0 0 5}$ & $\mathbf{0 . 2 0 0 9}$ & 27 & 0.1945 & 0.1945 \\
16 & 0.1785 & 0.1784 & 28 & 0.1365 & 0.1366 \\
18 & 0.1875 & 0.1876 & 29 & 0.0994 & 0.0995 \\
20 & 0.1061 & 0.1061 & & & \\
\hline
\end{tabular}

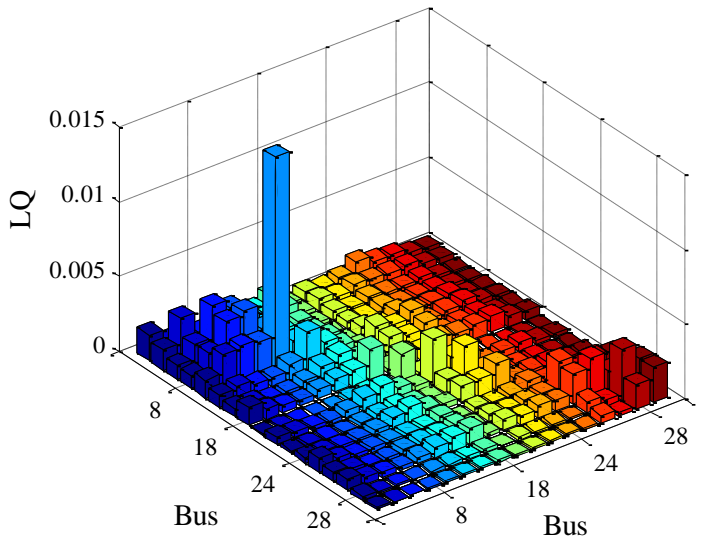

(a) $L-Q$ sensitivity matrix

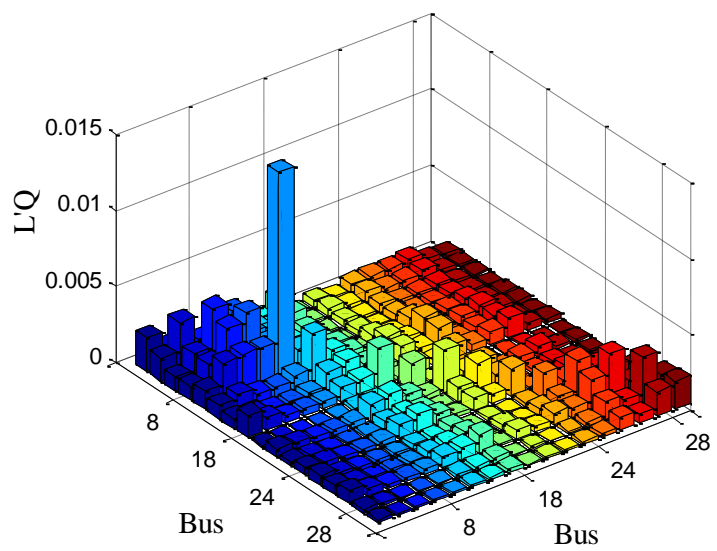

(b) $L^{\prime}-Q$ sensitivity matrix

Fig. 3. $L-Q$ and $L^{\prime}-Q$ sensitivity matrices for base load condition

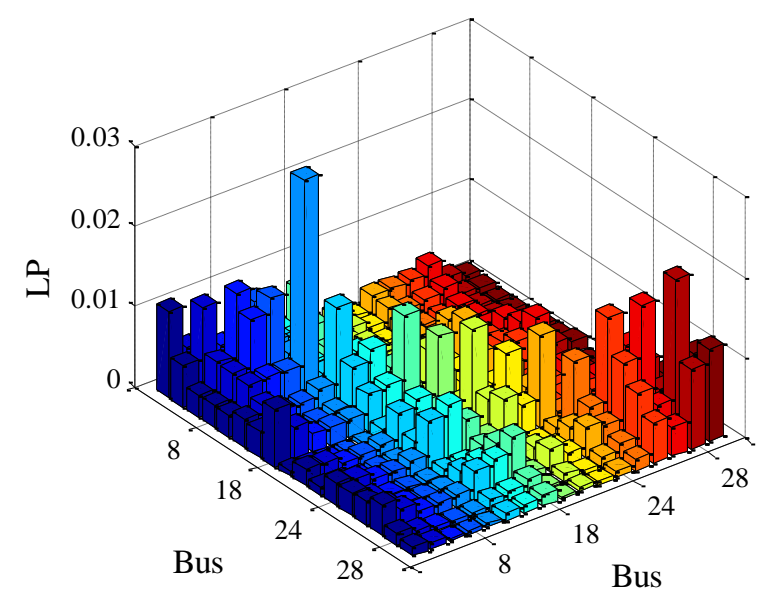

(a) $L-P$ sensitivity matrix

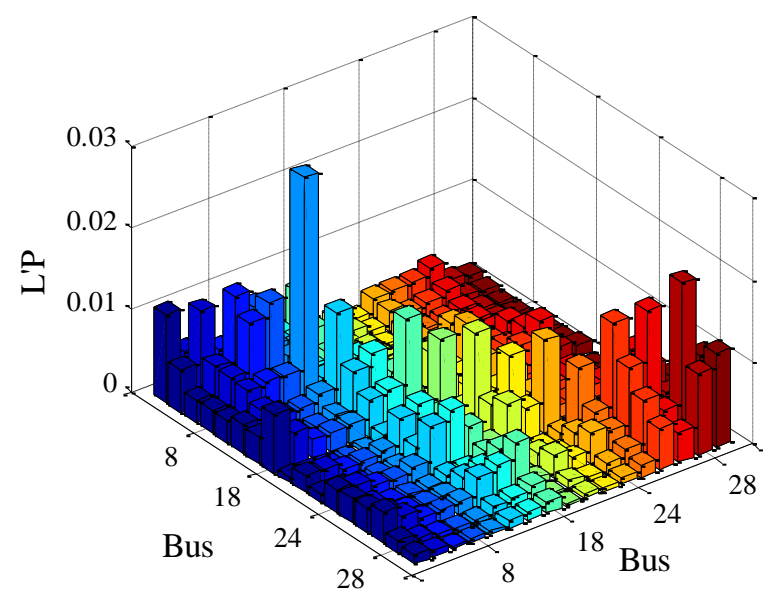

(b) $L^{\prime}-P$ sensitivity matrix

Fig. 4. $L-P$ and $L^{\prime}-P$ sensitivity matrices for base load condition

In TABLE 2, the $L-Q, L-P, L^{\prime}-Q$ and $L^{\prime}-P$ sensitivities of the critical bus, Bus 15 , are summarized. It is seen that the sensitivity of $L 15$ or $L^{\prime} 15$ to $Q 15$ or $P 15$ (in bold font) is much higher than the sensitivities of $L 15$ or $L^{\prime} 15$ to the reactive or real injection at other buses. It is also noticed that the sensitivity to Q16 or P16 is the second largest among the corresponding sensitivities. From the analytical expression of the sensitivity in (17), (18), (24), and (25) (especially in (24) and (25)), the numerical value of the sensitivity is closely related to the load level and the coupling impedance of the two locations. Bus 16 has a relatively high load level and is the nearest location to 15 among all the other load buses. This justifies the higher sensitivity of $L 15$ (or $L^{\prime} 15$ ) to Q16 and P16 over other 
locations.

Moreover, the sensitivities of $L 15$ and $L^{\prime} 15$ to reactive and real injection of Bus 15 (self-sensitivity) and Bus 16 (largest cross-sensitivity) are verified with details to demonstrate the correctness of the proposed sensitivities.

In order to verify the $L-Q$ sensitivities, the real power injections are kept constant. From the base load level, the $L$ (or $L^{\prime}$ ) indices of Bus 15 with a total reactive power reduction of 1.0 p.u. at 0.1 p.u. per step at the corresponding location (bus 15 or 16) are $a$ ) estimated by multiplying the sensitivity at the base condition with the reactive power reduction amount and $b$ ) calculated according to the updated power flow results with the reactive power change actually applied at the corresponding location. The $L$ (or $\left.L^{\prime}\right)$ indices estimated through $a$ ) are benchmarked with the actual indices calculated through $b$ ) at each step. This process is depicted in Fig. 5 where Fig. 5(a), (b), (c), and (d) correspond to the verification of $L-Q_{15,15}, L^{\prime}-Q_{15,15}, L-Q_{15,16}$, and $L^{\prime}-Q_{15,16}$ respectively. The errors are summarized in TABLE 3.

Table 2 Sensitivities of Bus 15 under Base Load Condition

\begin{tabular}{ccc||cc}
\hline Bus $\boldsymbol{i}$ & $\boldsymbol{L}-\boldsymbol{Q}_{15, \boldsymbol{i}}$ & $\boldsymbol{L}^{\prime}-\mathbf{Q}_{15, \boldsymbol{i}}$ & $\boldsymbol{L}_{\boldsymbol{P}} \boldsymbol{P}_{\mathbf{I 5 , \boldsymbol { i }}}$ & $\boldsymbol{L}^{\prime}-\boldsymbol{P}_{\mathbf{I 5 , i}}$ \\
\hline 3 & 0.0008 & 0.0009 & 0.0034 & 0.0033 \\
4 & 0.0009 & 0.0011 & 0.0042 & 0.0042 \\
7 & 0.0006 & 0.0007 & 0.0027 & 0.0027 \\
8 & 0.0006 & 0.0007 & 0.0027 & 0.0027 \\
12 & 0.0011 & 0.0009 & 0.0036 & 0.0036 \\
$\mathbf{1 5}$ & $\mathbf{0 . 0 0 2 7}$ & $\mathbf{0 . 0 0 3 2}$ & $\mathbf{0 . 0 1 2 7}$ & $\mathbf{0 . 0 1 2 6}$ \\
16 & 0.0017 & 0.0017 & 0.0066 & 0.0066 \\
18 & 0.0010 & 0.0011 & 0.0045 & 0.0044 \\
20 & 0.0004 & 0.0003 & 0.0013 & 0.0013 \\
21 & 0.0014 & 0.0012 & 0.0045 & 0.0045 \\
23 & 0.0010 & 0.0006 & 0.0023 & 0.0025 \\
24 & 0.0016 & 0.0015 & 0.0059 & 0.0059 \\
25 & 0.0005 & 0.0003 & 0.0012 & 0.0013 \\
26 & 0.0007 & 0.0006 & 0.0024 & 0.0024 \\
27 & 0.0009 & 0.0009 & 0.0037 & 0.0037 \\
28 & 0.0004 & 0.0003 & 0.0012 & 0.0012 \\
29 & 0.0004 & 0.0002 & 0.0008 & 0.0008 \\
\hline
\end{tabular}

According to Fig. 5(a) and (b), as the reactive power at Bus 15 increases, the errors of $L$ and $L^{\prime}$ indices first reduces to $0.0554 \%$ and $0.00495 \%$ and then increases monotonically to the maximum of $0.9301 \%$ and $1.1375 \%$ at 1.0 p.u. $Q$ reduction. In general, the sensitivities are accurate near the operating base but gain more errors as the variation increases. This is common in most sensitivity analyses that use linearized sensitivities to estimate the nonlinear power system equations. However, the errors of the proposed sensitivities at a relative large variation, 1.0 p.u., are still under $1.2 \%$ which demonstrates good accuracy. According to Fig. 5(c) and (d), the errors of estimated $L$ and $L^{\prime}$ indices increase monotonically to $1.2907 \%$ and $1.2935 \%$ respectively at 1.0 p.u. reactive reduction. Similarly, such errors can be considered small and demonstrate good accuracy of the proposed 
sensitivities. By comparing the errors in TABLE 3, it is concluded that in this case study 1) the sensitivities of the $L$ indices to reactive power bring less errors than the sensitivities to reactive power at other buses; 2) $L-Q$ sensitivities bring less errors compared with $L^{\prime}-Q$ sensitivities. 3) Both types of sensitivities achieve good accuracy in order to investigate the impact of reactive variation on voltage stability.

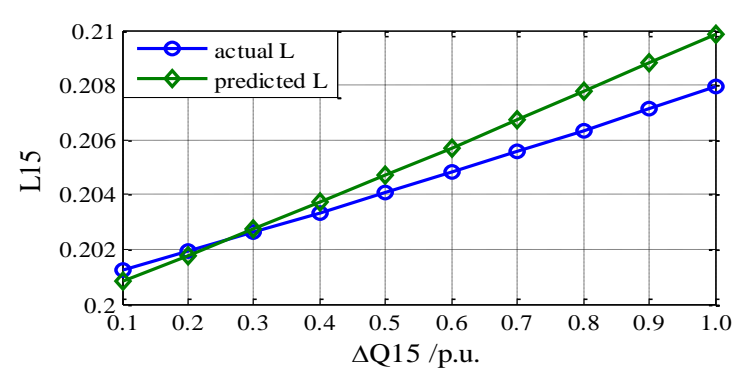

(a) $L-Q_{15,15}$

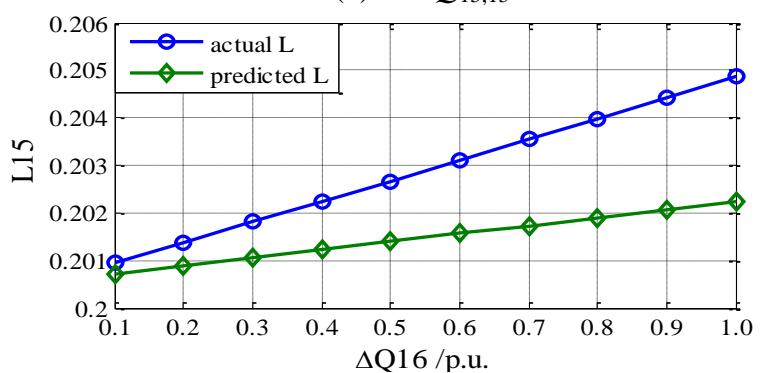

(c) $L-Q_{15,16}$

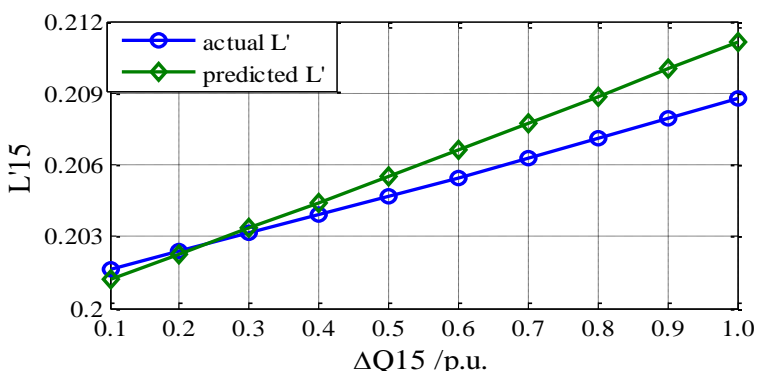

(b) $\quad L^{\prime}-Q_{15,15}$

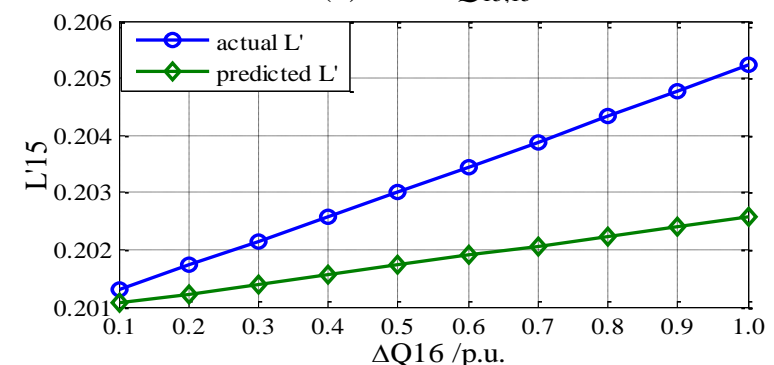

(d) $L^{\prime}-Q_{15,16}$

Fig. 5. Verification of $L-Q$ and $L^{\prime}-Q$ sensitivities of Bus 15

Table 3 Relative Error Of Predicted $L$ and $L^{\prime}$ based on $L-Q$ and $L^{\prime}-Q$ sensitivities

\begin{tabular}{lcccc}
\hline & \multicolumn{2}{c}{$\boldsymbol{L}-\boldsymbol{Q}_{\mathbf{1 5 , 1 5}}$} & \multicolumn{2}{c}{$\boldsymbol{L}-\boldsymbol{Q}_{\mathbf{1 5 , 1 6}}$} \\
\cline { 2 - 5 } /p.u. & $\begin{array}{c}\boldsymbol{L} \\
\text { prediction } \\
\text { error /\% }\end{array}$ & $\begin{array}{c}\boldsymbol{L}^{\prime} \\
\text { prediction } \\
\text { error /\% }\end{array}$ & $\begin{array}{c}\boldsymbol{L} \\
\text { prediction } \\
\text { error /\% }\end{array}$ & $\begin{array}{c}\boldsymbol{L}^{\prime} \\
\text { prediction } \\
\text { error /\% }\end{array}$ \\
\hline 0.1 & 0.2033 & 0.2033 & 0.1233 & 0.1238 \\
0.2 & 0.0734 & 0.0495 & 0.2479 & 0.2488 \\
0.3 & 0.0554 & 0.1032 & 0.3739 & 0.3751 \\
0.4 & 0.1833 & 0.2546 & 0.5010 & 0.5026 \\
0.5 & 0.3103 & 0.4049 & 0.6295 & 0.6313 \\
0.6 & 0.4363 & 0.5539 & 0.7592 & 0.7613 \\
0.7 & 0.5612 & 0.7017 & 0.8902 & 0.8926 \\
0.8 & 0.6852 & 0.8483 & 1.0225 & 1.0250 \\
0.9 & 0.8082 & 0.9935 & 1.1560 & 1.1587 \\
1.0 & 0.9301 & 1.1375 & 1.2907 & 1.2935 \\
\hline
\end{tabular}

Similar to the verification of $L-Q$ sensitivities, $L-P_{15,15}, L^{\prime}-P_{15,15}, L-P_{15,16}$, and $L^{\prime}-P_{15,16}$ are verified by benchmarking a) the $L$ and $L^{\prime}$ indices estimated by multiplying the sensitivity at the base condition with the real power reduction amount with b) $L$ and $L^{\prime}$ indices calculated according to the updated power flow results with the real power change actually applied at the corresponding location. This process is depicted in Fig. 6 where Fig. 6(a), (b), (c), and (d) correspond to the verification of $L-P_{15,15}, L^{\prime}-P_{15,15}, L-P_{15,16}$, and $L^{\prime}-P_{15,16}$ respectively. The errors are summarized in TABLE 4. According to Fig. 4, the sensitivities achieve good accuracy near the base 
operating but gain more errors as the variation increases. The errors of the proposed sensitivities at a relative large variation, 1.0 p.u., are still under $0.5 \%$ which demonstrates good accuracy. By comparing the errors in TABLE 4 , it is concluded that in this case study 1) the sensitivities of the $L$ indices to real power bring more errors than the sensitivities to real power at other buses; 2) Both types of sensitivities achieve good accuracy in order to investigate the impact of reactive variation on voltage stability; 3) $L-P$ sensitivities achieve better accuracy than $L-Q$ sensitivities.

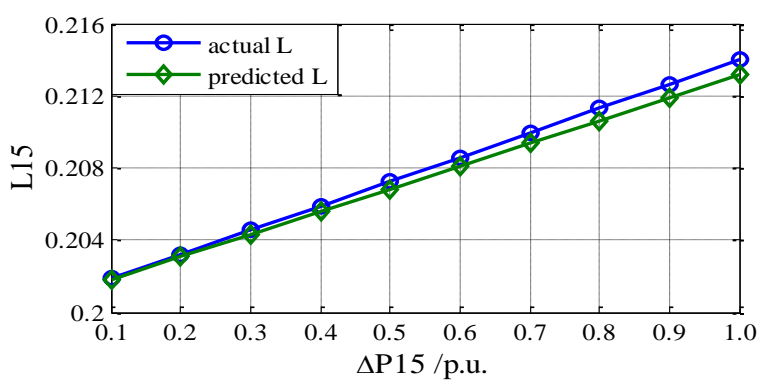

(a) $L-P_{15,15}$

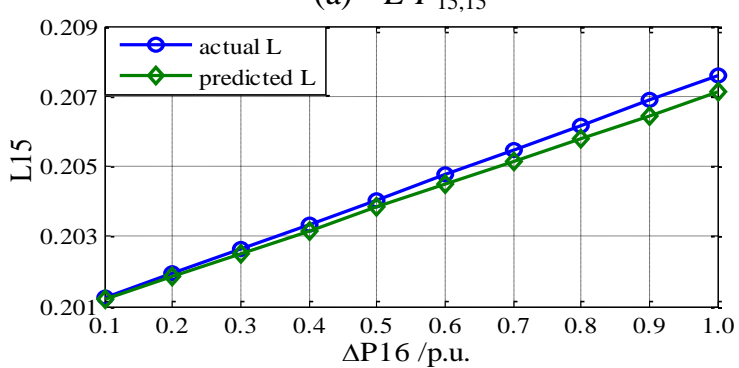

(c) $L-P_{15,16}$

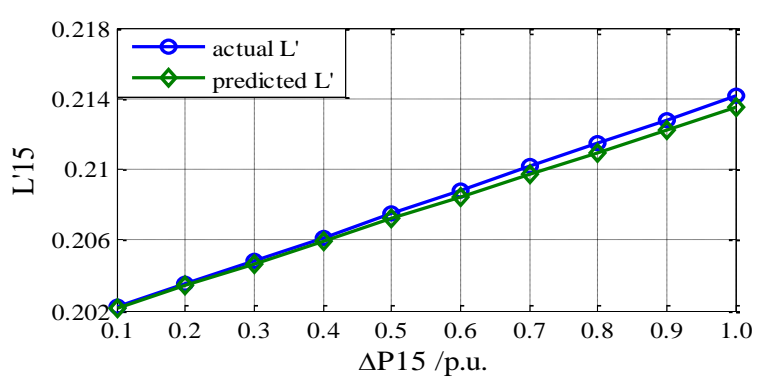

(b) $L^{\prime}-P_{15,15}$

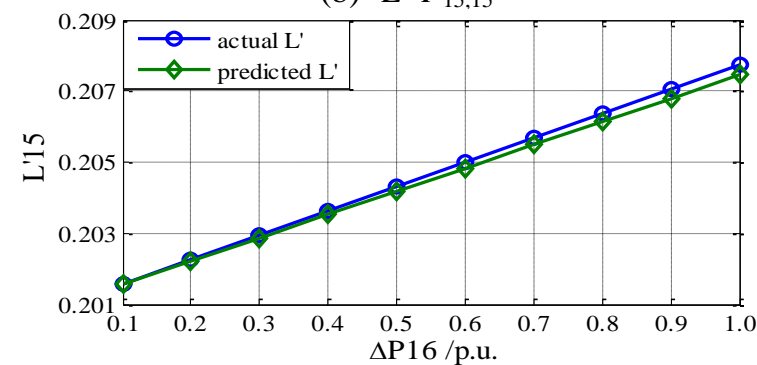

(d) $L^{\prime}-P_{15,16}$

Fig. 6. Verification of $L-P$ and $L^{\prime}-P$ sensitivities of Bus 15

Table 4 Relative Error of Predicted $L$ and $L^{\prime}$ based on $L-P$ and $L^{\prime}-P$ sensitivities

\begin{tabular}{lcccc}
\hline \multirow{\Delta}{*}{$\boldsymbol{P}$} & \multicolumn{2}{c}{$\boldsymbol{L}_{\mathbf{1 5 , 1 5}}$} & \multicolumn{2}{c}{$\boldsymbol{L}-\boldsymbol{P}_{\mathbf{1 5 , 1 6}}$} \\
\cline { 2 - 5 } /p.u. & $\begin{array}{c}\boldsymbol{L} \\
\text { prediction } \\
\text { error /\% }\end{array}$ & $\begin{array}{c}\boldsymbol{L}^{\prime} \\
\text { prediction } \\
\text { error /\% }\end{array}$ & $\begin{array}{c}\boldsymbol{L} \\
\text { prediction } \\
\text { error /\% }\end{array}$ & $\begin{array}{c}\boldsymbol{L}^{\prime} \\
\text { prediction } \\
\text { error /\% }\end{array}$ \\
\hline 0.1 & 0.0372 & 0.0261 & 0.0204 & 0.0110 \\
0.2 & 0.0754 & 0.0533 & 0.0416 & 0.0228 \\
0.3 & 0.1146 & 0.0815 & 0.0637 & 0.0354 \\
0.4 & 0.1549 & 0.1108 & 0.0866 & 0.0488 \\
0.5 & 0.1961 & 0.1412 & 0.1103 & 0.0631 \\
0.6 & 0.2383 & 0.1727 & 0.1348 & 0.0782 \\
0.7 & 0.2815 & 0.2052 & 0.1601 & 0.0941 \\
0.8 & 0.3256 & 0.2388 & 0.1862 & 0.1109 \\
0.9 & 0.3707 & 0.2734 & 0.2132 & 0.1285 \\
1.0 & 0.4168 & 0.3091 & 0.2410 & 0.1469 \\
\hline
\end{tabular}

\subsubsection{Verification of Proposed Voltage Stability Control}

In Section 2.3 and Section 3, a control strategy based on the proposed sensitivities is introduced. In this subsection, three scenarios of the 39-bus system are created in order to verify the applicability of the proposed sensitivities: 
Scenario 1: $0.64+\mathrm{j} 0.3$ p.u. load which is $20 \%$ of the original load is added to Bus 15 . Reactive power reserve at Bus 15 is 4p.u..

Scenario 2: 0.64+j 0.3 p.u. load is added to Bus 15. Reactive power reserves at Buses 15 and 16 are 2 p.u. and 3.5 p.u., respectively.

Scenario 3: $11+\mathrm{j} 5.335$ p.u. load which is $350 \%$ of the original load is added to Bus 15 . Branch $15-16$ is tripped creating an emergency condition.

In Scenarios 1 and 2, the control target, $L_{0}$, for the $L$ and $L^{\prime}$ indices is set as 0.2 which is the voltage stability level at the base load condition. In Scenario 3, which represents an emergency condition, the control target $L_{0}$ is set as 0.8 .

0.64+j 0.3 p.u. load is added to Bus 15 in first two scenarios which leads the $L$ and $L^{\prime}$ indices of Bus 15 , the critical bus, to 0.2113 and 0.2117 . The difference in the two scenarios is the reserve at Bus 15 .

\section{$\underline{\text { Scenario } 1}$}

After the load is added, $L$ and $L^{\prime}$ indices of load bus are increased shown in Step 0 in Fig. 7. The $L-Q$ and $L^{\prime}-Q$ sensitivities of Bus 15 are calculated and listed in TABLE 5. For both $L$ and $L^{\prime}$, the largest sensitivity is to $Q 15$ and the second largest one is to $Q 16$. Therefore, the first candidate location to apply compensation should be Bus 15 where the sensitivity is the largest. According to (26), with $L-Q_{15,15}=0.0028, L^{\prime}-Q_{15,15}=0.0034$, and $L_{0}=0.2$, the compensation to be applied is 3.9815 p.u. and 3.4637 p.u. respectively. Since the reserve at Bus 15 in this scenario is 4 p.u. which is larger than the calculated compensation amount, 3.9815 p.u. and 3.4637 p.u. reactive power are compensated at Bus 15 respectively. The $L$ and $L^{\prime}$ index, after the control is applied, are calculated based on the updated power flow results and are illustrated in Step 1 in Fig. 7. It is noted that after the compensations are applied, the $L$ index (Step 1 in Fig. 7(a)) and $L^{\prime}$ (Step 1 in Fig. 7(b)) index are successfully pulled down below $L_{0}$. In this scenario the sensitivities and the control strategy function properly with a good accuracy.

Table $5 L-Q$ and $L-Q^{\prime}$ Sensitivities of Bus 15 at Step 0

\begin{tabular}{ccc||ccc}
\hline $\begin{array}{c}\text { Bus No } \\
\text { of } \boldsymbol{Q}\end{array}$ & $\begin{array}{c}\boldsymbol{L}-\boldsymbol{Q} \\
\text { Sensitivities }\end{array}$ & $\begin{array}{c}\boldsymbol{L}^{\prime}-\mathbf{Q} \\
\text { Sensitivities }\end{array}$ & $\begin{array}{c}\text { Bus No } \\
\text { of } \boldsymbol{Q}\end{array}$ & $\begin{array}{c}\boldsymbol{L}-\boldsymbol{Q} \\
\text { Sensitivities }\end{array}$ & $\begin{array}{c}\boldsymbol{L}^{\prime}-\mathbf{Q} \\
\text { Sensitivities }\end{array}$ \\
\hline 3 & 0.0008 & 0.0009 & 21 & 0.0014 & 0.0012 \\
4 & 0.0010 & 0.0011 & 23 & 0.0010 & 0.0007 \\
7 & 0.0007 & 0.0007 & 24 & 0.0016 & 0.0016 \\
8 & 0.0007 & 0.0007 & 25 & 0.0005 & 0.0003 \\
12 & 0.0011 & 0.0010 & 26 & 0.0007 & 0.0006 \\
$\mathbf{1 5}$ & $\mathbf{0 . 0 0 2 8}$ & $\mathbf{0 . 0 0 3 4}$ & 27 & 0.0009 & 0.0010 \\
$\mathbf{1 6}$ & $\mathbf{0 . 0 0 1 8}$ & $\mathbf{0 . 0 0 1 7}$ & 28 & 0.0005 & 0.0003 \\
18 & 0.0011 & 0.0012 & 29 & 0.0004 & 0.0002 \\
20 & 0.0005 & 0.0004 & & & \\
\hline
\end{tabular}




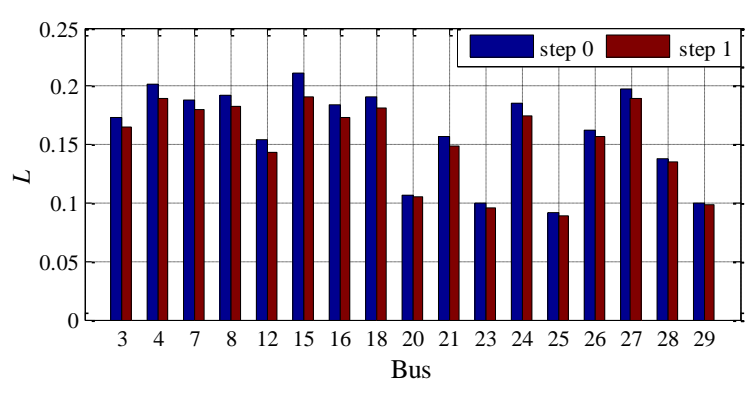

(a) Result based on $L-Q$ sensitivities

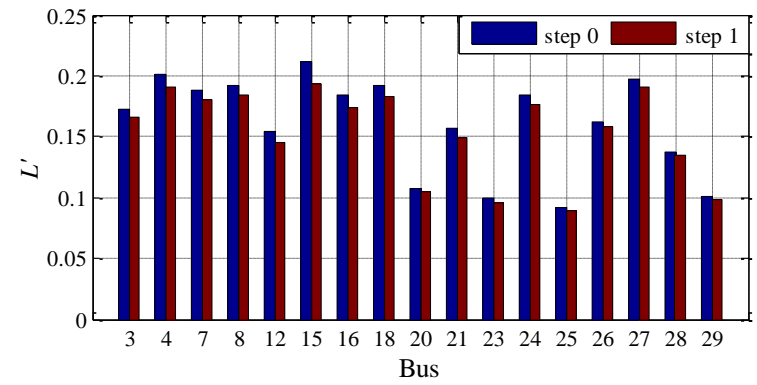

(b) Result based on $L^{\prime}-Q$ sensitivities

Fig. 7. Control results of Scenario 1, 39 bus system

\section{$\underline{\text { Scenario } 2}$}

The same perturbation is applied in this scenario and therefore the $L-Q$ and $L^{\prime}-Q$ sensitivities of Bus 15 are the same as Scenario 1, which is shown in TABLE 5. However, the reserve at Bus 15 is reduced to 2.0 p.u. which is less than the required compensation 3.9815 p.u. and 3.4637 p.u calculated by (26). Therefore the 2.0 reserve is first applied in step 1. In the second step, further compensation at Bus 16, the location with the second largest sensitivities, should be applied. According to (27), with $L-Q_{15,16}=0.0018$ and $L^{\prime}-Q_{15,16}=0.0017$, the compensations at Bus 16 are 3.1944 p.u. and 2.8202 p. u. respectively. Since the calculated compensations at Bus 16 are less than the reactive power reserve at Bus 16, the calculated compensations are then applied at Bus 16. The results are illustrated by Step 2 in Fig. 8. The actual $L$ and $L^{\prime}$ indices for Bus 15 after Step 2 are 0.1906 and 0.1915 respectively, both under $L_{0}$. It is therefore demonstrated that the sensitivities and controls work properly in this scenario.

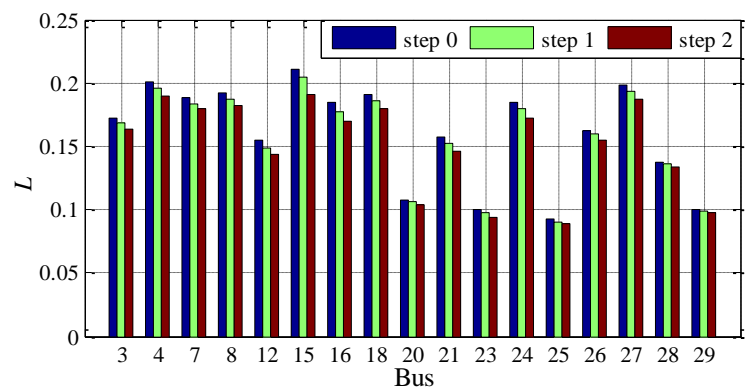

(a) Results based on $L-Q$ sensitivities

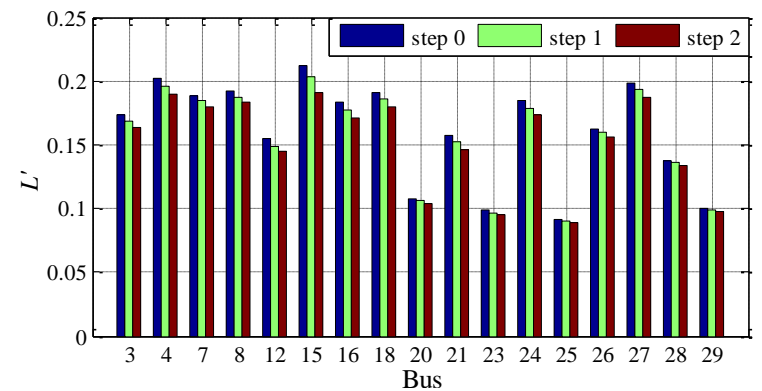

(b) Results based on $L^{\prime}-Q$ sensitivities

Fig. 8. Control results of Scenario 2, 39 bus system

\section{$\underline{\text { Scenario } 3}$}

In this scenario, the load at Bus 15 is increased by $350 \%$ of its original load. The $L$ and $L^{\prime}$ indices of all load buses after the load increasing are depicted in Fig. 9 (a) and (b) respectively labelled as "Step 0" where the $L$ and $L^{\prime}$ indices of Bus 15 increased to 0.4482 and 0.4521 respectively. Following the load perturbation, Branch 15-16 is tripped. "Step 1" in Fig. 9 (a) and (b) illustrates the $L$ and $L$ ' indices after this outage respectively, where the $L$ and $L^{\prime}$ indices of Bus 15 surged to 0.9778 and 0.9816 . With the $L$ indices close to 1 , the system at Step 1 is considered voltage insecure and emergency control action, load shedding in the proposed control strategy should be applied. 
The $L-Q, L-P, L^{\prime}-Q$ and $L^{\prime}-P$ sensitivities are calculated based on the system condition on Step 1 and are listed in Table 6. With $L_{0}$ set as 0.8 , the amount of load shedding at Bus 15 is determined through (28) is $3.3622++\mathrm{j} 1.6076$ p.u. by $L-Q$ and $L-P$ sensitivities and $2.9010+\mathrm{j} 1.3871$ p.u. by $L^{\prime}-Q$ and $L^{\prime}-P$ sensitivities. The calculated amount of load shedding is applied and the results are presented by "Step 2" in Fig. 9 (a) and (b) respectively. It is seen that the $L$ index of Bus 15 after the load shedding is 0.8122 and the $L^{\prime}$ index of Bus 15 after load shedding is 0.7787 . The proposed sensitivities and control strategy successfully pulls the system to a stable operating point under this emergency condition.

Table $6 L-P, L^{\prime}-P L-Q$ and $L^{\prime}-Q$ Sensitivities of Step 1

\begin{tabular}{ccccc||ccccc}
\hline Bus & $\boldsymbol{L}-\boldsymbol{P}$ & $\boldsymbol{L}^{\prime}-\boldsymbol{P}$ & $\boldsymbol{L}-\boldsymbol{Q}$ & $\boldsymbol{L}^{\prime}-\mathbf{Q}$ & Bus No & $\boldsymbol{L}-\boldsymbol{P}$ & $\boldsymbol{L}^{\prime}-\boldsymbol{P}$ & $\boldsymbol{L}-\boldsymbol{Q}$ & $\boldsymbol{L}^{\prime}-\mathbf{Q}$ \\
\hline 3 & 0.0050 & 0.0061 & 0.0045 & 0.0027 & 21 & 0.0008 & 0.0011 & 0.0010 & 0.0005 \\
4 & 0.0137 & 0.0160 & 0.0109 & 0.0071 & 23 & 0.0004 & 0.0006 & 0.0006 & 0.0003 \\
7 & 0.0086 & 0.0105 & 0.0076 & 0.0047 & 24 & 0.0011 & 0.0015 & 0.0013 & 0.0007 \\
8 & 0.0085 & 0.0103 & 0.0073 & 0.0046 & 25 & 0.0010 & 0.0017 & 0.0015 & 0.0007 \\
12 & 0.0123 & 0.0153 & 0.0114 & 0.0068 & 26 & 0.0011 & 0.0016 & 0.0014 & 0.0007 \\
$\mathbf{1 5}$ & $\mathbf{0 . 0 4 4 1}$ & $\mathbf{0 . 0 4 0 8}$ & $\mathbf{0 . 0 2 7 8}$ & $\mathbf{0 . 0 3 5 9}$ & 27 & 0.0016 & 0.0021 & 0.0017 & 0.0010 \\
16 & 0.0012 & 0.0017 & 0.0014 & 0.0007 & 28 & 0.0005 & 0.0008 & 0.0008 & 0.0004 \\
18 & 0.0031 & 0.0040 & 0.0031 & 0.0018 & 29 & 0.0003 & 0.0006 & 0.0005 & 0.0003 \\
20 & 0.0002 & 0.0003 & 0.0003 & 0.0001 & & & & &
\end{tabular}

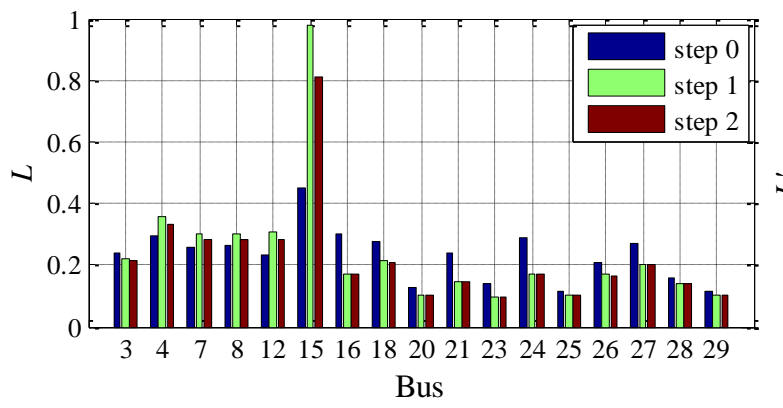

(a) Results based on $L-P, L-Q$ sensitivities

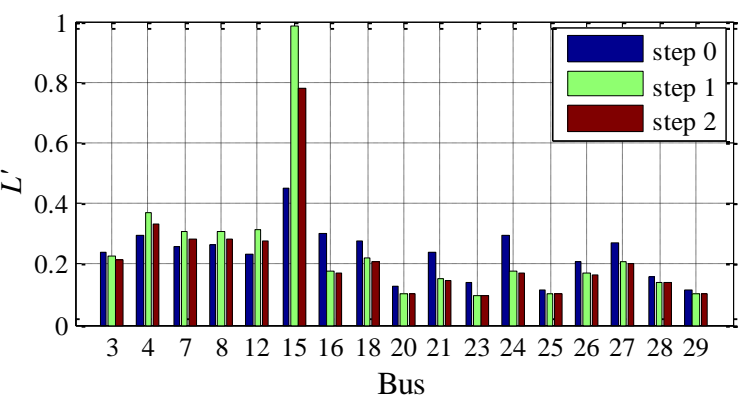

(b) Results based on $L^{\prime}-P, L^{\prime}-Q$ sensitivities

Fig. 9. Control results of Scenario 3, 39 bus system

\subsection{IEEE118 bus System}

The proposed sensitivities and control strategy are further tested on the IEEE 118 bus system [36]. Two scenarios are considered representing a normal operating condition and an emergency condition respectively.

\section{$\underline{\text { Scenario } 1}$}

In this scenario, all the loads are proportionally increased by $200 \%$ which represents a normal operating condition. The $L$ and $L^{\prime}$ indices after the load increasing are depicted in Fig. 10 (a) and (b) respectively. The critical bus turns out to be Bus 44 with an $L$ index of 0.2840 and an $L^{\prime}$ index of 0.2773 . With $L_{0}$ set to 0.2 , the $L-Q$ and $L^{\prime}-Q$ sensitivities are calculated accordingly and the reactive compensations are determined by (26) to be 1.57 p.u. and 1.39 p.u. respectively. After the compensation, the $L$ and $L^{\prime}$ indices of Bus 15 are reduced to 0.2279 and 0.2164 respectively which are illustrated in Fig. 10 (c) and (d). 


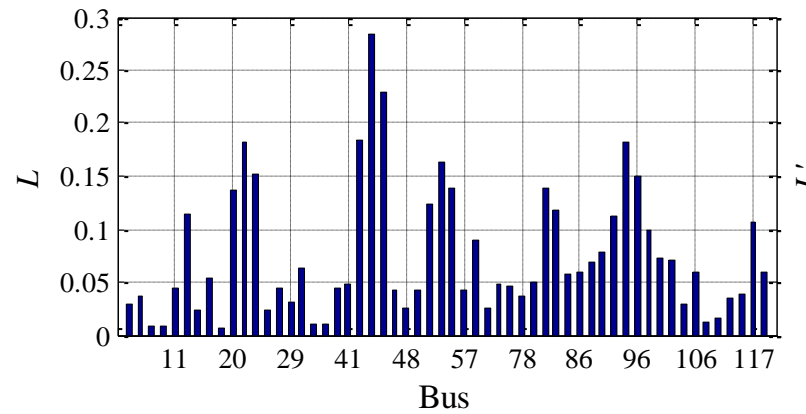

(a) $L$ indices after load increasing

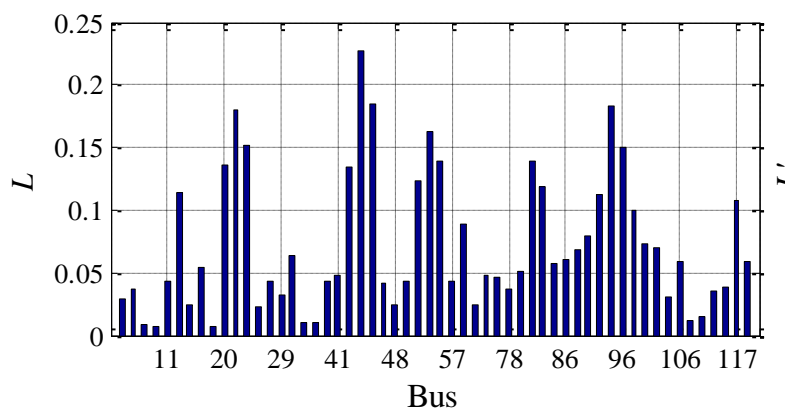

(c) Result based on $L-Q$ sensitivities

Fig. 10. Control results of Scenario 1, 118 bus system.

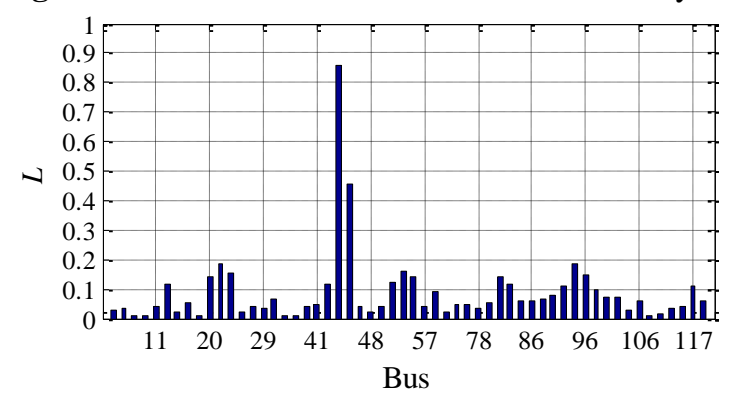

(a) $L$ indices after branch outage

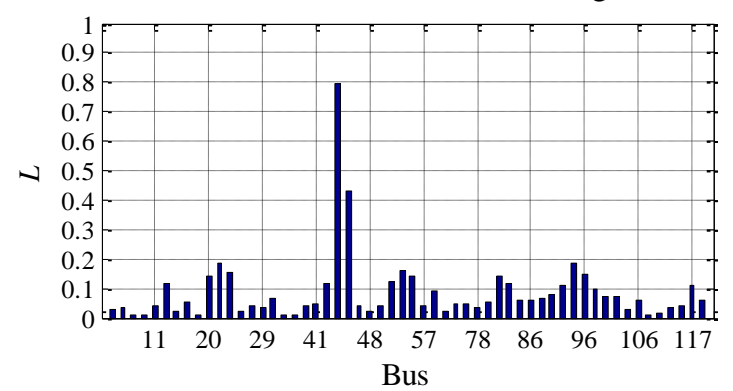

(c) Result based on $L-Q, L-P$ sensitivities

Fig. 11. Control results of Scenario 2, 118 bus system.

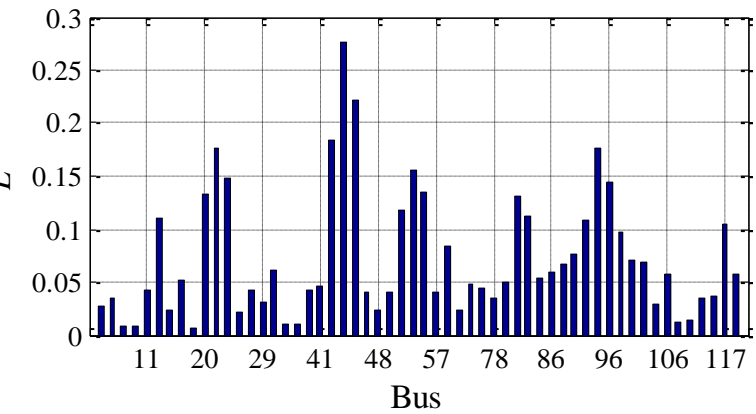

(b) $L^{\prime}$ indices after load increasing

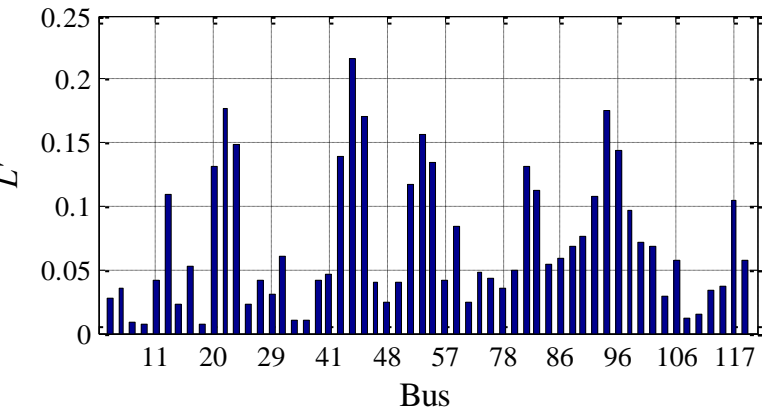

(d) Result based on $L^{\prime}-Q$ sensitivities

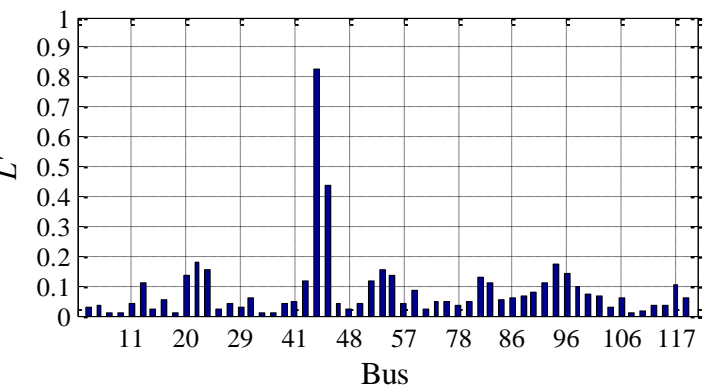

(b) $L^{\prime}$ indices after branch outage

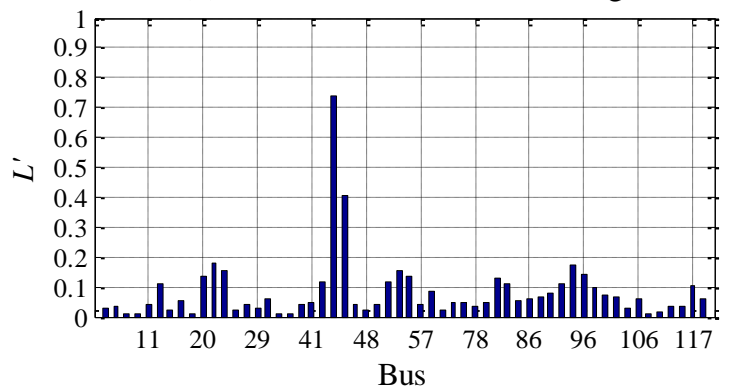

(d) Result based on $L^{\prime}-Q, L^{\prime}-P$ sensitivities

\section{Scenario 2}

In this scenario, the load at Bus 44 is proportionally increased by $281 \%$ which raises the $L$ and $L^{\prime}$ index of Bus 44 to 0.5087 and 0.4970 . Following the load increase, Branch $43-44$ is tripped. The $L$ and $L^{\prime}$ indices of all load buses after the branch outage are depicted in Fig. 11 (a) and (b). It is noted that the $L$ and $L^{\prime}$ index of Bus 44 reach 0.8615 and 0.8287 which are both greater than the $L_{0}$ of 0.8 under emergency condition. Therefore, emergency load shedding should be applied. The sensitivities are calculated and the load shedding at Bus 44 is determined 
according to (28) with $L_{0}$ set to 0.8 . The load to be shed is $0.0947+\mathrm{j} 0.0888$ p.u. according to $L-P$ and $L-Q$ sensitivities or $0.0881+$ j0.0826 p.u. according to $L^{\prime}-P$ and $L^{\prime}-Q$ sensitivities. After the load shedding is applied, the $L$ and $L^{\prime}$ indices are shown in Fig. 11 (c) and (d). The $L$ and $L^{\prime}$ index of Bus 44 drops to 0.7939 and 0.7413 respectively and are both below the 0.8 threshold.

It is demonstrated that in both scenarios of the 118 bus system, the proposed sensitivities and control strategy perform effectively.

\section{CONCLUSION}

This work proposes wide-area measurement-based voltage stability sensitivities, $L-Q$ and $L-P$ sensitivities, based on the $L$ index, an existing voltage stability index. Moreover, taking advantage of the characteristics of a high-voltage transmission network, a simplified $L^{\prime}$ index as well as $L^{\prime}-Q$ and $L^{\prime}-P$ sensitivities are proposed which greatly reduce the computational costs and makes the proposed sensitivity practical on large scale power systems. Based on the proposed sensitivities, an effective yet simple control strategy for improving the voltage stability is proposed. Case studies on the New England 39-bus system and the IEEE 118 bus system demonstrate that the proposed $L-Q, L-P, L^{\prime}-Q$, and $L^{\prime}-P$ sensitivities provide reasonable and quantitative analyses of the coupling impact of nodal reactive injection to bus voltage stability; the proposed voltage stability control strategy yields effective and accurate control actions to improve the system-level voltage stability and has great potential to be implemented online due to its simplicity; the proposed sensitivities, as well as the control strategy, can equip the system operators with multidimensional information regarding the system status.

\section{ACKNOWLEDGEMENT}

The authors would like to acknowledge the support in part by National Natural Science Foundation of China (No.51377016, No.51477027, 51677022, 51607033 and 51607034), Northeast Dianli University Research Project (No.BSJXM-201501), and the CURENT research center funded by US NSF and DOE under the NSF grant EEC-1041877.

\section{References}

[1] Mahmud N, Zahedi A. Review of control strategies for voltage regulation of the smart distribution network with high penetration of renewable distributed generation. Renewable and Sustainable Energy Reviews $2016 ; 64: 582-595$.

[2] Esmaili M, Shayanfar H, Amjady N. Multi-objective congestion management incorporating voltage and transient stabilities. Energy 2009; 34:1401-1412. 
[3] Wang D, Parkinson S, Miao W, Jia H, Crawford C, Djilali N. Online voltage security assessment considering comfort-constrained demand response control of distributed heat pump systems. Applied Energy 2012; 96:104-114.

[4] Londero RR, Affonso CM, Vieira JPA. Long-Term Voltage Stability Analysis of Variable Speed Wind Generators. IEEE Trans Power Syst. 2014; 29(12):439-447.

[5] Modarresi J, Gholipour E, Khodabakhshian A. A comprehensive review of the voltage stability indices. Renewable and Sustainable Energy Reviews 2016; 63:1-12.

[6] Sarimuthu C, Ramachandaramurthy V, Agileswari K, Mokhlis H. A review on voltage control methods using on-load tap changer transformers for networks with renewable energy sources. Renewable and Sustainable Energy Reviews 2016; 62:1154-1161.

[7] Kim M, Park J, Nam Y. Market-clearing for pricing system security based on voltage stability criteria. Energy $2011 ; 36: 1255-1264$.

[8] Esmaili M, Shayanfar H, Amjady N. Multi-objective congestion management incorporating voltage and transient stabilities. Energy 2009; 34:1401-1412.

[9] Ashrafi A. Shahrtash SM. Dynamic Wide Area Voltage Control Strategy Based on Organized Multi-Agent System. IEEE Trans Power Syst 2014; 29(17):2590-2601.

[10] Jiang T, Bai L, Jia H, Yuan H, Li F. Identification of voltage stability critical injection region in bulk power systems based on the relative gain of voltage coupling. IET Gener Transm Distrib 2016; 10(18):1495-1503.

[11] Wang Y, Pordanjani I, Li W, Xu W, Chen T. Vaahedi E, Gurne J. Voltage stability monitoring based on the concept of coupled single-port circuit. IEEE Trans Power Syst 2011; 26(17):2154-2163.

[12] Yuan H, Jiang T, Jia H, Li F, et al. Real-Time Wide-area Loading Margin Sensitivity (WALMS) in Power Systems, in Proc. IEEE PES General Meeting, Denver, CO, USA, Jul 2015.

[13] Canizares C. A. Voltage stability assessment: concepts, practices and tools. IEEE PES Power System Stability Subcommittee Special Publication; 2002.

[14] Hammad A. E, Kuehn W. A. Computation Algorithm for Assessing Voltage Stability at AC/DC Interconnection. IEEE Trans Power Syst 1986; 1(12):209-215.

[15] Chiang H. D, Wang C. S, Flueck A.J. Look-ahead voltage and load margin contingency selection functions for large-scale power systems. IEEE Trans Power Syst 1997; 12(12):173-180.

[16] Gao B, Morison G.K, Kundur P. Voltage stability evaluation using modal analysis. IEEE Trans Power Syst 1992; 7(15):1529-1542.

[17] Tamura Y, Sakamoto K, Tayama Y. Voltage instability proximity index (VIPI) based on multiple load flow solutions in ill-conditioned power systems. in: Proceedings of the 27th IEEE Conference on Decision and Control, 1988.

[18] Vournas C. D. Voltage Stability and Controllability Indices for Multimachine Power Systems. IEEE Trans Power Syst 1995; 10(14):1183-1194.

[19] Nagao T, Tanaka K, Takenaka K. Development of Static and Simulation Programs for Voltage Stability Studies of Bulk Power System. IEEE Trans Power Syst 1997; 12(12):273-281.

[20] Yorino N, Harada S, Cheng H. A Method to Approximate a Closest Loadability Limit Using Multiple Load Flow Solutions. IEEE Trans Power Syst 1997; 12(12):424-429.

[21] Milosevic B, Begovic M. Voltage-stability protection and control using a wide-area network of phasor measurements. IEEE Trans Power Syst 2003; 18(12):121-127. 
[22] Salehi V, Mohammed O. Real-time voltage stability monitoring and evaluation using synchorophasors, in Proc. North Amer. Power Symp. (NAPS), Aug 2011.

[23] Diao R, Sun K, Vittal V, et al. Decision tree based online voltage security assessment using PMU measurements, IEEE Trans Power Syst 2009; 24(13):832-839.

[24] Moghavvemi M, Omar FM. Technique for contingency monitoring and voltage collapse prediction. IEE Proc. Gener. Transm. Distrib. 1998; 145(17):634-640.

[25] Smon I, Verbic G, Gubina F. Local voltage-stability index using Tellegen's theorem. IEEE Trans Power Syst 2006; 21(14):1267-1275.

[26] Sodhi R, Srivastava S, Singh S. A simple scheme for wide area detection of impending voltage instability. IEEE Trans Smart Grid 2012; 3(13):818-827.

[27] Gong Y, Schulz N, Guzman A. Synchrophasor-based real time voltage stability index, in Proc. IEEE PES PSCE2006, Atlanta, October 2006:1029-1036

[28] Kessel P, Glavitsch H. Estimating the Voltage Stability of a Power System. IEEE Trans Power Delivery 1986; 1(14):346-354.

[29] Jia H, Yu X, Yu Y. An improved voltage stability index and its application. Int J Elect Power Energy Syst 2005; 27(19):567-574.

[30] Yuan H, Li F. Hybrid Voltage Stability Assessment (VSA) for N-1 Contingency. Electric Power Systems Research 2015; 122:65-75.

[31] Abdelkader S. M, Morrow D.J. Online Tracking of Thevenin Equivalent Parameters Using PMU Measurements. IEEE Trans Power Syst 2012; 27(13):975-983.

[32] Corsi S, Taranto GN. A real-time voltage instability identification algorithm based on local phasor measurements. IEEE Trans Power Syst 2008; 23(14):1271-1279.

[33] Liu J-H, Chu C-C. Wide-Area Measurement-Based Voltage Stability Indicators by Modified Coupled Single-Port Models. IEEE Trans Power Syst 2014; 29(13):756-764.

[34] Wang Y, Wang C-S, Lin F, et al. Incorporating Generator Equivalent Model into Voltage Stability Analysis. IEEE Trans Power Syst 2013; 28(15):4857-4866.

[35] Yuan H, Li F. A Comparative Study of Measurement-Based Thevenin Equivalents Identification Methods. in Proc. 46th North American Power Symposium (NAPS), Pullman, WA, Sept 2014.

[36] Power System Test Case Archive. http://www.ee.washington.edu/research/pstca/. 